\title{
Modelo de Calidad para la Gestión de las TIC en el Proceso de Enseñanza para Instituciones Educativas Peruanas del Nivel Secundario
}

\section{RESUMEN}

Debido a la importancia de elevar el rendimiento académico de los alumnos de la educación básica regular del nivel secundario, el trabajo que se presenta en este artículo se desarrolla en el marco de Los Sistemas de Calidad para la Gestión de las TIC. Se desarrolla un Modelo de Calidad para la Gestión de las TIC., para instituciones educativas peruanas del nivel secundario. El modelo está basado en el Modelo del Ciclo de Mejora Continua de W. Deming (Planificar, Hacer, Verificar y Actuar - PDCA) y la Norma de Autoevaluación ISO 9004.2009. Asimismo considera de forma integral los procesos y estrategias de enseñanza en las Instituciones Educativas y el modelo educativo del país. A la vez, se presenta una guía de implementación, el que permite seguir apropiadamente los procedimientos para realizar el diagnóstico de la situación actual de la institución educativa, y las actividades de mejora o mantenimiento que propone el modelo.

Palabras clave: calidad total, modelo de excelencia de malcolm baldrige, modelo efqm, ISO 9004-2009, pdca

QUALITY MODEL FOR THE MANAGEMENT OF ICT in the teaching process for Peruvian EDUCATIONAL INSTITUTIONS AT THE SECONDARY LEVEL

\section{ABSTRACT}

Due to the importance of raising the academic performance of students in the regular basic education at the secondary level, the work presented in this paper is developed in the framework of Quality Systems for the Management of ICT. The model has developed an implementation guide that allows properly follow procedures for diagnosis and improvement activities. It is based on the Model Cycle Demming Continuous Improvement (PDCA) and the Self-Assessment Standard ISO 9004.2009 also comprehensively considers the processes and strategies of teaching in educational institutions and the educational model of the country.

The planning and implementation of self-assessment will reveal what level of maturity is the institution with respect to the management of ICT, to finally act through improvement activities seeking to correct nonconformities to reach the level of maturity No. 1 or the next level of maturity.

Keywords: excellence model malcolm baldrige, efam model, ISO 9004-2009, pdca, quality improvement

\section{INTRODUCCIÓN}

Un modelo de calidad es un conjunto de prácticas vinculadas a los procesos de gestión y el desarrollo de proyectos [4], con los modelos de calidad las organizaciones logran alcanzar un impacto estratégico que cumpla con los objetivos fijados para mejorar la calidad del servicio $[1,2,5,6,12,13,15]$. La filosofía nace en Japón partiendo del concepto de la calidad del producto [7].

El modelo desarrollado en el presente trabajo está basado en el modelo de calidad de W. Deming denominado Ciclo de Mejora Continua (PDCA) [5, 9] y en la Norma ISO 9004-2009 [8] para el proceso de autoevaluación [8]. Tanto el modelo como la Norma han sido articulados para lograr la gestión de calidad de las TIC en los procesos de enseñanza, de instituciones educativas peruanas de educación secundaria. A la vez que se desarrolla el modelo se presenta una guía de implementación, el cual describe los componentes y actividades que se deben seguir para aplicarlo.

El Modelo de calidad busca mejorar el desempeño de las instituciones educativas peruanas de educación secundaria en la gestión de las TIC, para de esta manera elevar el rendimiento académico de los alumnos en su proceso de aprendizaje. Pueden participar todas las áreas a excepción del área de Educación Física. Y los procesos de la Institución Educativa a considerar son: Gestión de la Dirección, Gestión de Recursos y el Pedagógico.

El modelo desarrollado podrá ser aplicado únicamente para instituciones educativas peruanas con una infraestructura tecnológica adecuada en un marco educativo por competencias.

La Guía Metodológica es un instrumento práctico para la implementación del Modelo de calidad para la gestión de las TIC, contiene los procedimientos necesarios para evaluar a una institución educativa y determinar el nivel de madurez en que se encuentra. Con los resultados obtenidos de la autoevaluación se procede a realizar las actividades de mejora o mantenimiento en las áreas y criterios que se requieran.

La estructura del resto del presente artículo es como sigue: en la Sección 2 se presenta el Modelo de Calidad para la Gestión de las TICs desarrollado; en la Sección 3 se describe el proceso

\footnotetext{
* Magíster en Ingeniería de Sistemas. Docente MINEDU. Jenny_victoria75@hotmail.com

** Doctora en Informática. Docente Asociada-FISI-UNMSM. nlasernap@unmsm.edu.pe
} 
de autoevaluación; en 4 se detalla el contenido de la guía de implementación, en 5 se presentan las conclusiones del trabajo realizado, y Finalmente en 6 se muestran las referencias bibliográficas utilizadas.

\section{MODELO DE CALIDAD PARA LA GESTIÓN DE LAS TIC}

El modelo desarrollado busca mejorar el desempeño de las instituciones educativas peruanas de educación secundaria. Tiene como objetivo principal elevar el rendimiento académico de los alumnos y así contribuir a lograr las expectativas trazadas en el sector educación. El modelo se basa en el ciclo de mejora continua de W. Deming (PDCA) [5, 9], que tiene como componentes: Planificar $(P)$, Hacer (D), Verificar (C) y Actuar (A). Razón por la cual el modelo que se desarrolla en este trabajo consta de cuatro componentes: Planificación (P), Diagnóstico (D), Verificación (V) y las actividades (A), tal como se puede observar en la Figura 1.

El componente Planificación, establece desarrollar un plan del proyecto para la gestión de calidad de las TIC de una institución educativa peruana de nivel secundario. Mientras que, el componente Diagnóstico, consiste en realizar el proceso de autoevaluación en base a la Norma Internacional de Calidad ISO 9004-2009 [8] a la Institución Educativa seleccionada, considerando como puntos principales la gestión pedagógica, la gestión institucional y los aprendizajes esperados en los alumnos. Este componente se explica con mayor detalle en la sección 3 de este artículo.

El componente Verificar, dispone recopilar los datos obtenidos en el componente Diagnóstico para analizar los resultados y conocer el nivel de madurez de gestión de las TIC alcanzado por la institución. Los niveles de madurez según la Norma pueden tomar valores de 1 a 5 . Entretanto, el componente Actividades, permite realizar el conjunto de actividades del plan de mejora o mantenimiento para las áreas seleccionadas de la institución que lo requieran.

\section{DESCRIPCIÓN DE LA HERRAMIENTA DE AU- TOEVALUACIÓN}

La herramienta se basa en la Norma ISO 9004 2009 [8], que incluye dos tablas de autoevaluación, las cuales son: a) De los elementos claves, y b) Para los elementos detallados. Ambas tablas utilizan cinco niveles de madurez (del 1 al 5) que son aplicados a las áreas que guardan relación directa con el uso de las TIC. Tal y como se observan en las Tablas 1 y 2 respectivamente.

La Tabla de Autoevaluación para los elementos claves, permite evaluar los criterios de la tabla para medir el desempeño actual de la institución para la gestión de las TIC, y determinar el nivel de madurez en que se encuentra.

Los criterios que se evalúan de los elementos claves son: la Gestión, el Liderazgo, la Estrategia y Política, los Recursos, los Procesos, los Resultados, el Seguimiento y Medición, la Mejora, innovación y aprendizaje.

La Tabla de Autoevaluación para los elementos detallados, evalua el desempeño actual de los elementos claves de la institución para la gestión de las TIC, pero lo hace de manera detallada, utilizando una tabla para cada elemento clave.

Figura 1. Modelo de Calidad para la Gestión de las TIC basado en la Norma ISO $9004-2009$ y del Modelo PDCA Fuente: [Moreno, 2014]

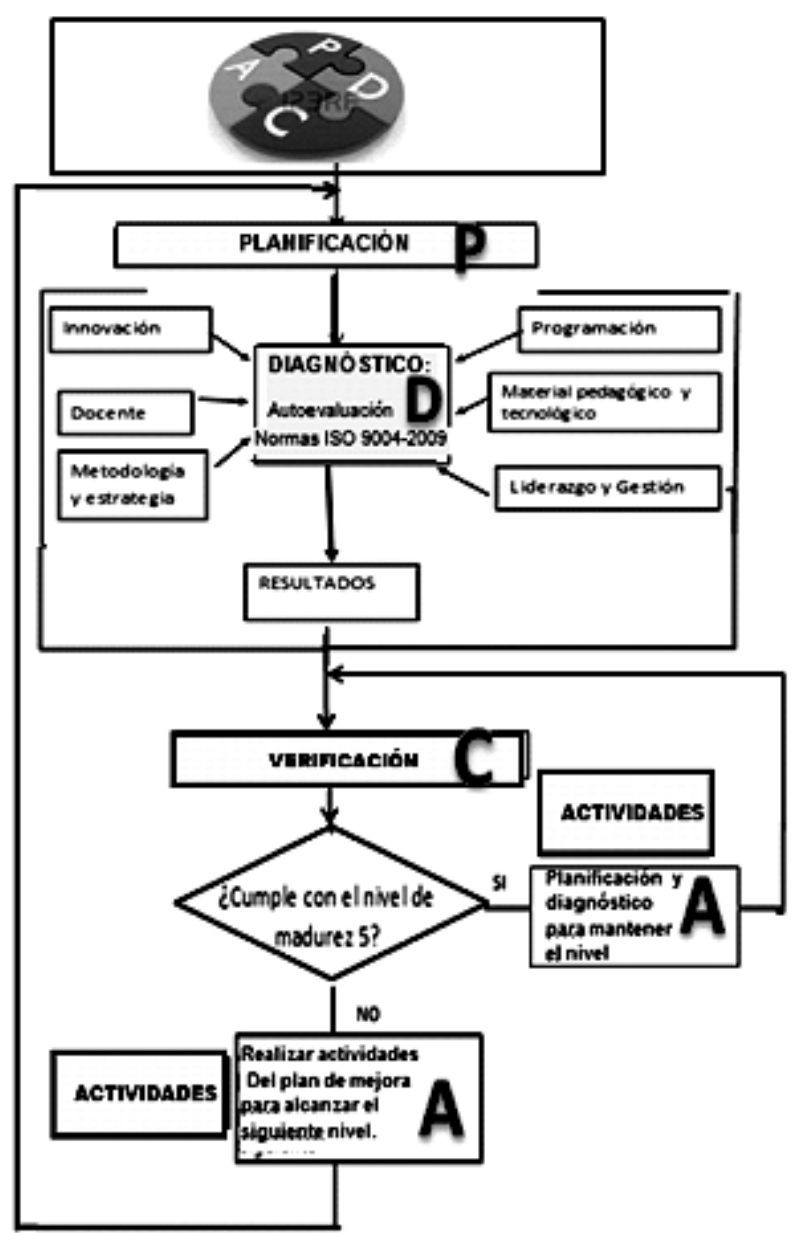


Tabla 1. Autoevaluación para los elementos Claves [8]

\begin{tabular}{|c|c|c|c|c|c|}
\hline \multirow{2}{*}{ ELEMENTOS CLAVES: Criterios } & \multicolumn{5}{|c|}{ Niveles de madurez } \\
\hline & 1 & 2 & 3 & 4 & 5 \\
\hline ¿Cuál es el centro de interés de la dirección? & & & & & \\
\hline ¿Cuál es el enfoque del liderazgo? (Gestión) & & & & & \\
\hline ¿Cómo decidimos qué es importante? (Estrategia y Política) & & & & & \\
\hline ¿Qué necesitamos para obtener resultados? (Recursos) & & & & & \\
\hline ¿Cómo se organizan las actividades? (Procesos) & & & & & \\
\hline ¿Cómo se logran los resultados? (Seguimiento y Medición) & & & & & \\
\hline $\begin{array}{l}\text { ¿Cómo se realiza el seguimiento de los resultados? (Seguimiento y } \\
\text { Medición) }\end{array}$ & & & & & \\
\hline $\begin{array}{l}\text { ¿Cómo se deciden las prioridades de mejora? (Mejora, Innovación y } \\
\text { aprendizaje) }\end{array}$ & & & & & \\
\hline ¿Cómo tiene lugar el aprendizaje? (Mejora, Innovación y aprendizaje) & & & & & \\
\hline
\end{tabular}

Tabla 2. Autoevaluación para los elementos detallados [8]

\begin{tabular}{|c|c|c|c|c|c|c|}
\hline \multirow{2}{*}{$\begin{array}{l}\text { ELEMENTOS } \\
\text { DETALLADOS }\end{array}$} & \multirow{2}{*}{ CRITERIOS } & \multicolumn{5}{|c|}{ Niveles de madurez } \\
\hline & & 1 & 2 & 3 & 4 & 5 \\
\hline \multirow{4}{*}{$\begin{array}{l}\text { Gestión para el Éxito } \\
\text { Sostenido de una Institución } \\
\text { Educativa }\end{array}$} & $\begin{array}{l}\text { Gestión para el éxito sostenido. } \\
\text { Generalidades }\end{array}$ & & & & & \\
\hline & Éxito Sostenido & & & & & \\
\hline & Entorno de la Institución & & & & & \\
\hline & $\begin{array}{l}\text { Partes Interesadas, necesidades y } \\
\text { expectativas }\end{array}$ & & & & & \\
\hline \multirow{4}{*}{ Estrategia y Política } & Estrategia y política. Generalidades & & & & & \\
\hline & Formulación de la estrategia y política. & & & & & \\
\hline & Despliegue de la estrategia y política. & & & & & \\
\hline & Comunicación de la estrategia y política. & & & & & \\
\hline \multirow{2}{*}{ Gestión de los Recursos } & Gestión de los recursos. Generalidades & & & & & \\
\hline & Personal de la Institución Educativa & & & & & \\
\hline Gestión de los Procesos & $\begin{array}{l}\text { Planificación y control de los procesos. } \\
\text { Generalidades }\end{array}$ & & & & & \\
\hline \multirow{2}{*}{$\begin{array}{l}\text { Seguimiento, Medición, } \\
\text { Análisis y Revisión }\end{array}$} & $\begin{array}{l}\text { Seguimiento, Medición, Análisis y } \\
\text { Revisión. Generalidades }\end{array}$ & & & & & \\
\hline & $\begin{array}{l}\text { Medición con respecto a los indicadores } \\
\text { claves de desempeño }\end{array}$ & & & & & \\
\hline \multirow{4}{*}{$\begin{array}{l}\text { Mejora Innovación y } \\
\text { Aprendizaje }\end{array}$} & Innovación y Aprendizaje. Generalidades & & & & & \\
\hline & Mejora & & & & & \\
\hline & Innovación & & & & & \\
\hline & Aprendizaje & & & & & \\
\hline
\end{tabular}




\subsection{Autoevaluación de los elementos claves}

En esta tabla se desarrollan nueve criterios: 1) El centro de interés de la dirección, 2) El enfoque del liderazgo, 3) Que es necesario para obtener resultados utilizando las TIC, 4) Gestión de los recursos tecnológicos, 5) Organización de las actividades de los procesos pedagógicos, 6) Acerca de los resultados a nivel institucional, 7) Seguimiento de los resultados del aprendizaje, 8) Procesos de mejora, innovación y aprendizaje con prioridad, 9) El lugar del aprendizaje.

Para cada criterio se especifican los indicadores para cada uno de los cinco niveles de madurez. Los valores de los indicadores permiten definir en que nivel de madurez se encuentra un criterio.

En la Tabla 3 se desarrollan solo cuatro de los nueve criterios, en la tesis [Moreno, 2014] Modelo de Calidad para la Gestión de las TIC en el Proceso de enseñanza, se especifican detalladamente los 9 criterios que intervienen. Por ejemplo, en el criterio 1, ¿Cómo es el centro de interés de la dirección?, los indicadores para el nivel de madurez 1 son: a) Planifica en el planAnual y en las unidades didácticas insertar las TIC, b) Considera las opiniones de los padres de familia en la planificación del plan anual.

Para medir el nivel de madurez en el que se encuentra un colegio secundario, se aplican encuestas a los docentes, directivos y padres de familia. Tal como se describen en la sección 4 del presente artículo.

\subsection{Autoevaluación de los Elementos Detallados.}

Se desarrolla una tabla para cada uno los siguientes elementos claves: 1) Gestión para el éxito sostenido, 2) Estrategia y política, 3) Gestión de los recursos tecnológicos, 4) Gestión de los procesos pedagógicos, 5) Seguimiento, medición, Análisis y revisión de los resultados del aprendizaje, 6) Procesos de mejora, innovación y aprendizaje con prioridad.
En cada tabla se especifican los criterios a evaluar, y para cada criterio se establecen los indicadores para cada uno de los cinco niveles de madurez.

Igualmente, para medir el nivel de madurez en el que se encuentra un colegio secundario, se aplican encuestas a los docentes, directivos y padres de familia para ver el nivel de cumplimiento de los criterios.

En las Tablas 4 y 5 se desarrollan los elementos detallados para: 1) Gestión para el éxito sostenido de una Institución Educativa, y 2) Estrategia y política, respectivamente. $\mathrm{Y}$ en cada tabla se presentan como ejemplos solamente 2 criterios. En la tesis [Moreno, 2014], se especifican detalladamente las 6 tablas de autoevaluación de elementos detallados, y todos los criterios que intervienen.

\subsection{Restricciones del Modelo}

El Modelo de Calidad para la Gestión de las TIC's en el Proceso de Enseñanza para Instituciones Educativas Peruanas del Nivel Secundario, presenta las siguientes restricciones:

- Las instituciones educativas deberán de contar con una infraestructura tecnológica adecuada que permita que todas las aulas puedan utilizar estos recursos.

- Los docentes previamente deben de tener conocimientos mínimos en: Ofimática, cómo crear un correo electrónico y cómo inscribirse y utilizar la Plataforma PerúEduca.

- No se considera la gestión administrativa de la institución educativa.

- Debe utilizarse únicamente para el nivel secundario de la educación básica regular, no puede ser utilizado para otras modalidades o Institutos porque la estructura del modelo corresponde a los procesos y capacidades del nivel secundario. 


\begin{tabular}{|c|c|c|c|c|c|}
\hline \multirow{5}{*}{ 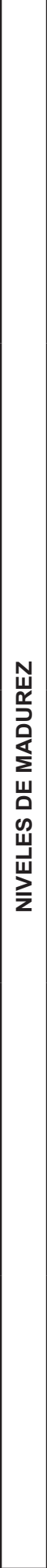 } & $\frac{n}{\stackrel{0}{\Phi}}$ & 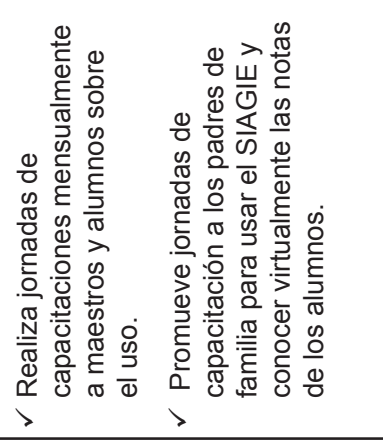 & 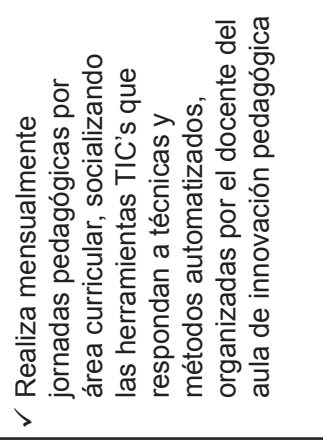 & 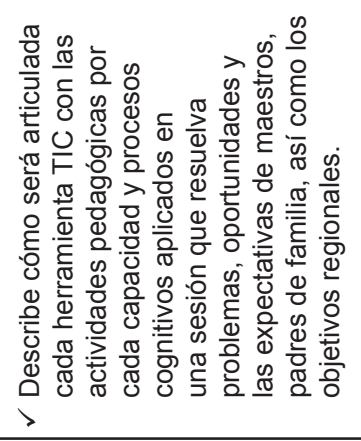 & 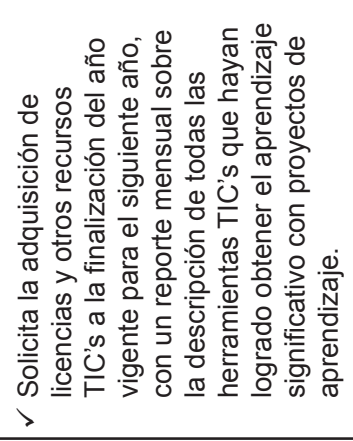 \\
\hline & $\frac{\bar{d}}{\stackrel{\nabla}{\mathbf{z}}}$ & 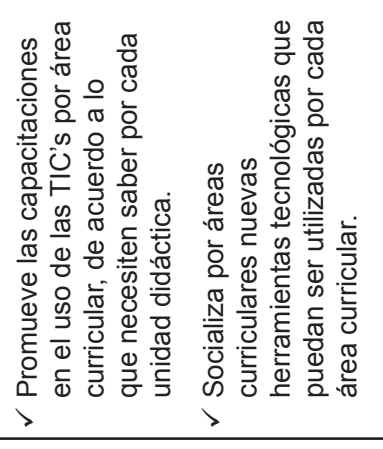 & 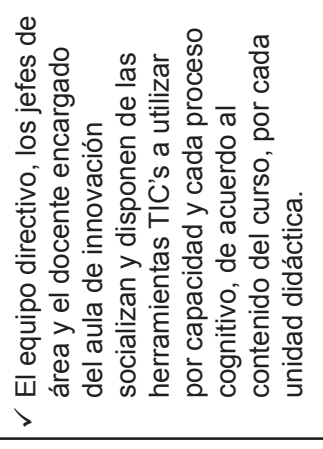 & 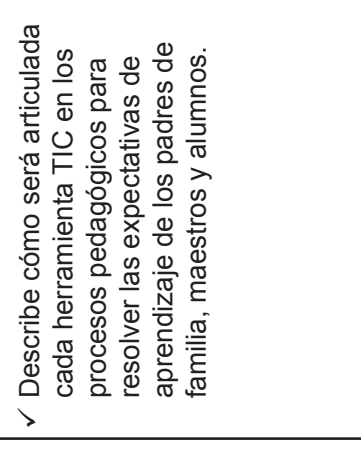 & 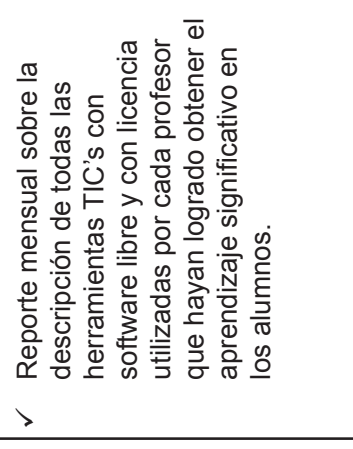 \\
\hline & $\frac{m}{\frac{m}{z}}$ & 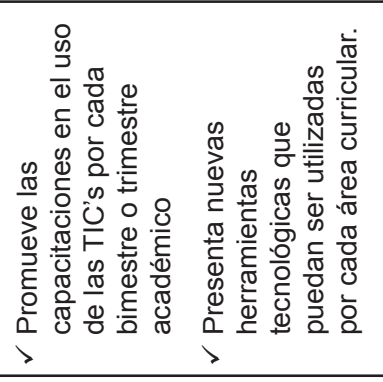 & 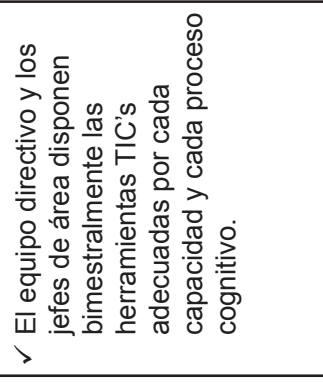 & 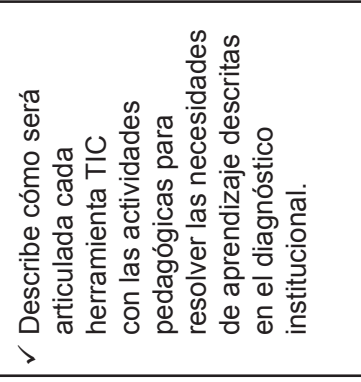 & 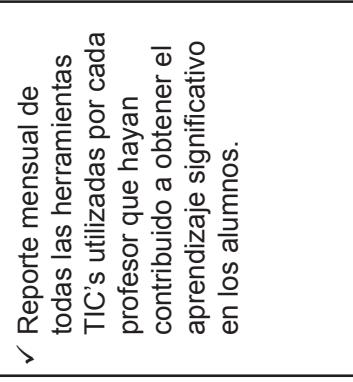 \\
\hline & $\frac{N}{\stackrel{N}{z}}$ & 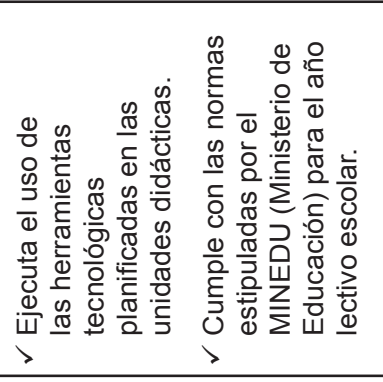 & 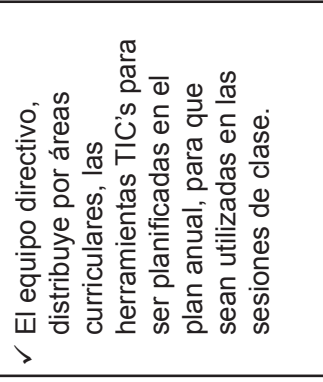 & 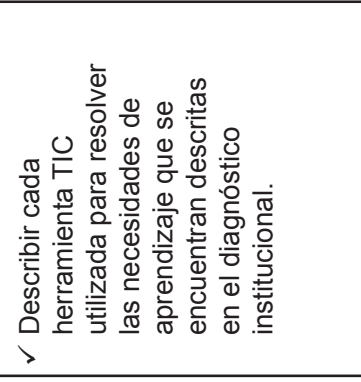 & 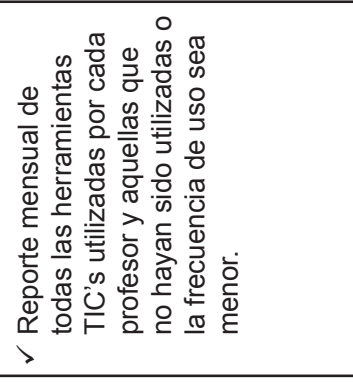 \\
\hline & $\overline{\bar{d}} \overline{\bar{z}}$ & 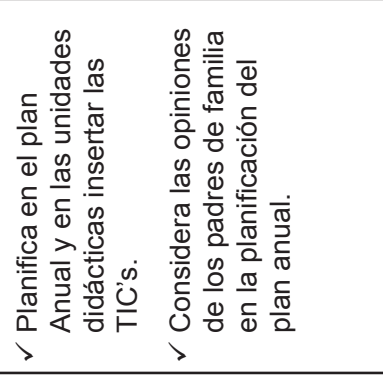 & 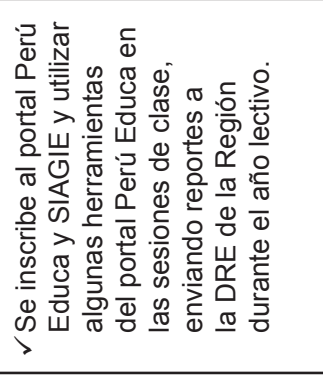 & 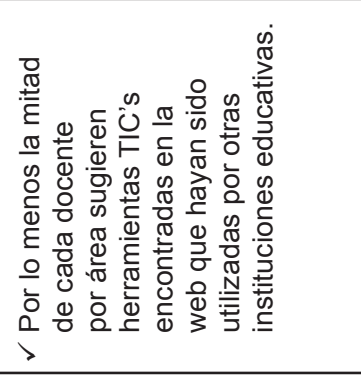 & 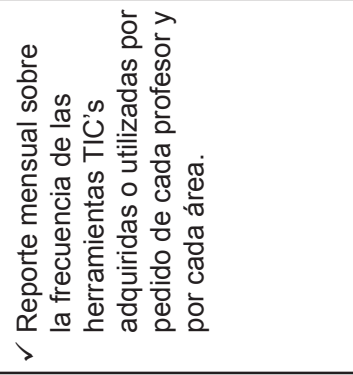 \\
\hline 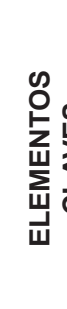 & 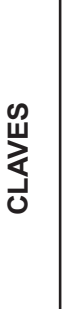 & 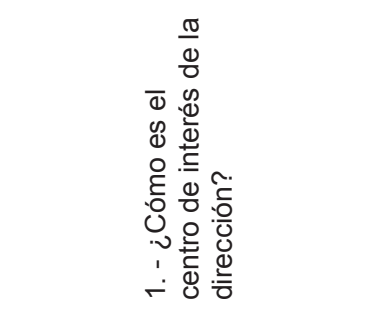 & 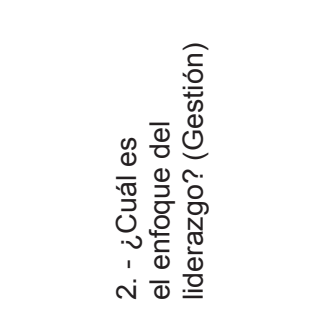 & 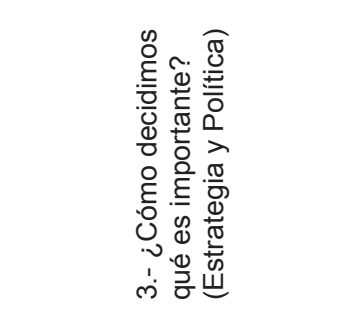 & 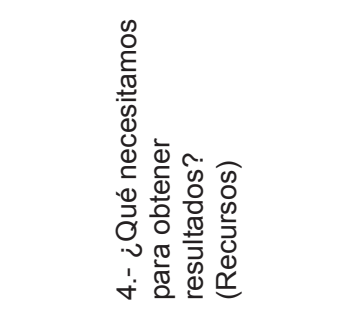 \\
\hline
\end{tabular}




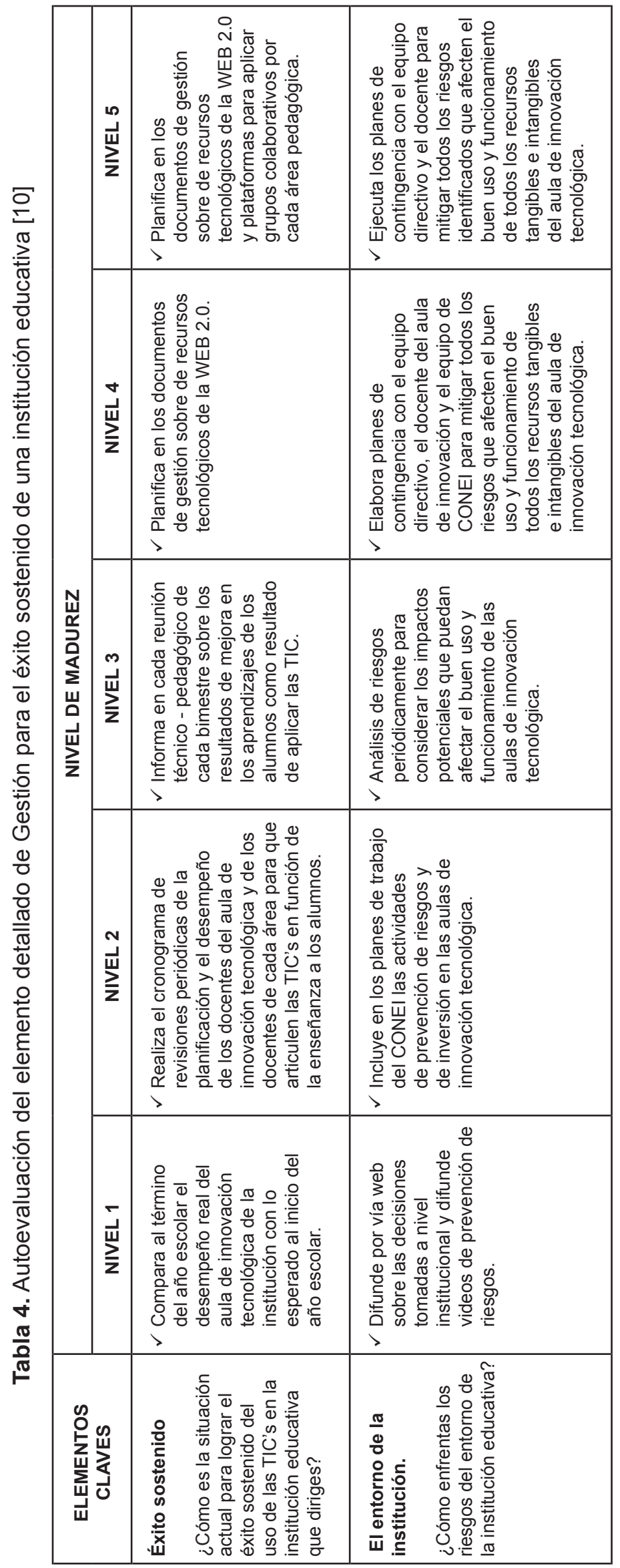

\begin{tabular}{|c|c|c|c|}
\hline \multirow{5}{*}{ 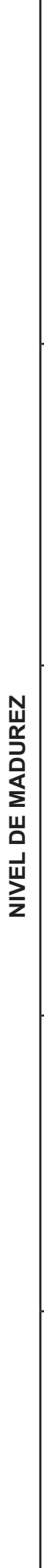 } & $\sum_{\bar{z}}^{\stackrel{n}{\Psi}}$ & 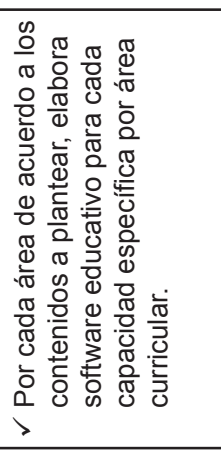 & 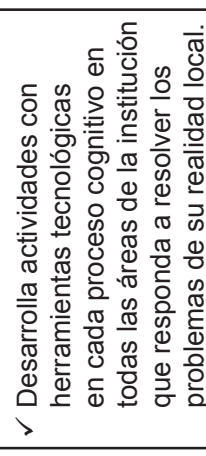 \\
\hline & $\sum_{\bar{z}}^{\vec{U}}$ & 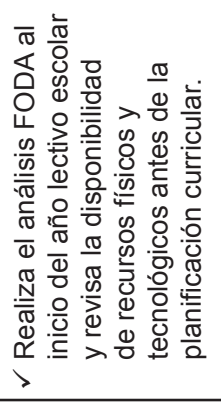 & 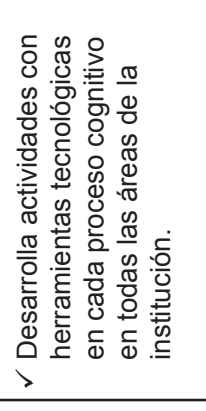 \\
\hline & $\sum_{\bar{z}}^{m}$ & 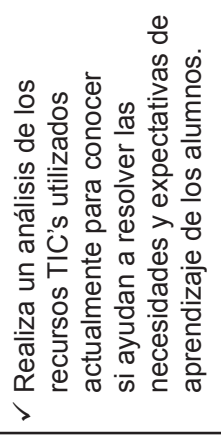 & 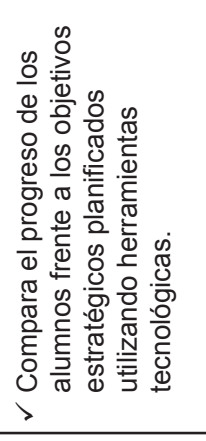 \\
\hline & $\sum_{z}^{N}$ & 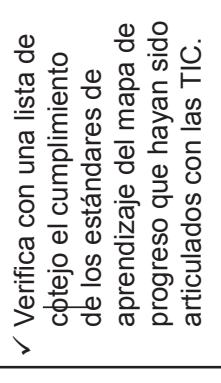 & 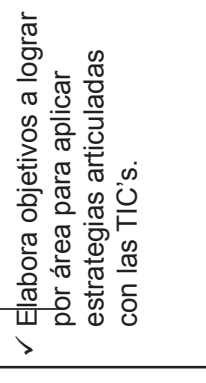 \\
\hline & $\sum_{\bar{z}}^{\bar{\Psi}}$ & 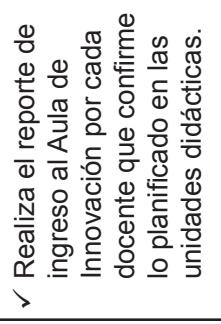 & 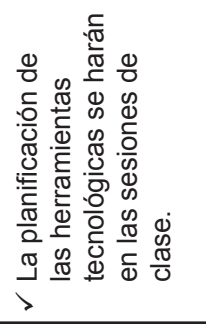 \\
\hline 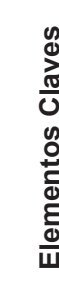 & & 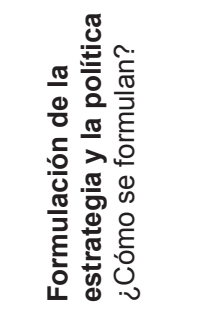 & 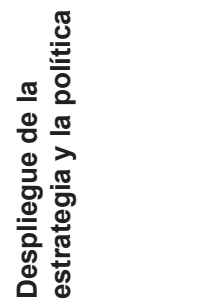 \\
\hline
\end{tabular}




\section{GUÍA METODOLÓGICA DEL MODELO}

La Guía Metodológica es un instrumento práctico para la implementación del Modelo de calidad para la gestión de las TIC, contiene los procedimientos necesarios para evaluar a una institución educativa y determinar el nivel de madurez en que se encuentra. Con los resultados obtenidos de la autoevaluación se procederá a realizar las actividades de mejora en las áreas y criterios que se requieran. El modelo se aplica a instituciones educativas públicas del nivel secundario que cuenten con infraestructura tecnológica implementada.

\subsection{Alcance de la Guía Metodológica}

a. La presente guía es de fácil implementación y muy práctica para el ámbito educativo, sobre todo en el área de Innovación Pedagógica, que muchas veces los recursos disponibles no están siendo utilizados de manera coherente con el enfoque curricular, los recursos se utilizan sin tomar en cuenta que pueden ser de mayor utilidad en determinados momentos pedagógicos o áreas curriculares.

b. La flexibilidad del modelo permite adaptarlo a cualquier institución educativa que tenga implementada una infraestructura tecnológica adecuada, porque los indicadores de los niveles de madurez de cada criterio están basados con el nuevo enfoque curricular del país.

c. Es necesario cumplir con todos los indicadores de los criterios de cada nivel para poder pasar al siguiente nivel, y en el caso de que algunos indicadores se encuentren en niveles más avanzados, entonces se debe de hacer un seguimiento para mantenerlos.

\subsection{Descripción de la Guía Metodológica}

La guía metodológica describe las actividades que se deben realizar para cada componente que conforman el modelo.

En el componente de Planificación establece desarrollar un plan del proyecto para la gestión de calidad de las TIC de una institución educativa peruana de nivel secundario. En el componente de Diagnóstico se realizará el proceso de autoevaluación que consiste en elaborar las tablas de autoevaluación y la elaboración del cuestionario.

En el componente Verificar, se analizan los resultados obtenidos en el componente Diagnóstico y se conoce el nivel de madurez de gestión de las TICs alcanzado por la institución. el componente Actividades, se realizan los planes de mejora o mantenimiento para las áreas seleccionadas de la institución que lo requieran.

A continuación, se describen las actividades que están incluidas en los componentes del modelo propuesto que siguen la secuencia que se muestra en la Figura 2.

Figura 2. Secuencia de las actividades de los componentes en la Guía metodológica.

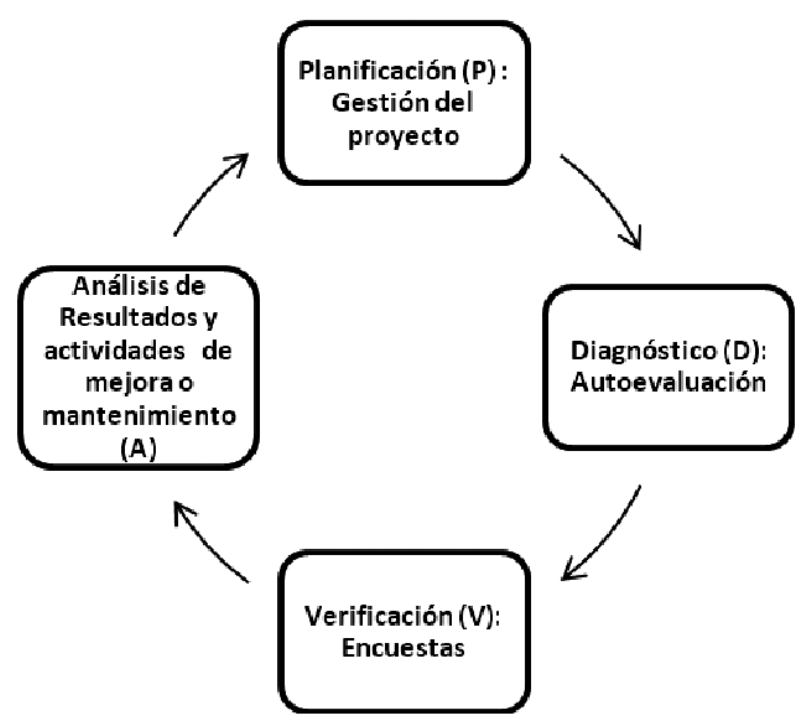

\subsubsection{Planificación (P): Gestión del Proyecto}

Se tomará en cuenta las siguientes pautas.

- Nombrar al equipo del proyecto. Estará conformado por el Director, el subdirector de Formación General, los jefes de cada área curricular y el docente del Aula de Innovación Tecnológica.

- Designar al jefe del proyecto. Del equipo de docentes que conforman el proyecto, será escogido el responsable del proyecto, tomando en cuenta que debe conocer acerca del modelo de gestión de la calidad así como el uso y manejo pedagógico de los recursos tecnológicos.

- El jefe del proyecto elaborará el plan del proyecto. En donde se establecerán los objetivos del proyecto, se especificará quienes conforman el equipo directivo, se detallará quienes son los jefes de cada área curricular, se establecerán los tiempos que se requerirán para llevar a cabo las actividades del proyecto, y otros aspectos concernientes a la organización y control del desarrollo del proyecto.

- El equipo directivo asignará conjuntamente con el Jefe del proyecto (podría ser el Docente 
del Aula de Innovación Tecnológica-DAIP), las responsabilidades de cada docente que conforman el equipo del proyecto para que se realice cada procedimiento del Modelo.

- El equipo del proyecto realizará un análisis situacional con respecto al buen uso de las TIC mediante la Autoevaluación.

- El jefe del proyecto capacitará a los docentes acerca del Modelo de Gestión de las TIC, que se incluirá dentro de las jornadas de reuniones técnico-pedagógico del Proyecto Educativo Institucional, PEI.

\subsubsection{Diagnóstico (D): Autoevaluación}

En esta fase, el equipo del proyecto participará en la elaboración de los indicadores que corresponden a los criterios de cada tabla de autoevaluación.

\subsubsection{Procedimiento para la Autoevaluación [3, 11]}

a. Establecer los requisitos de la evaluación:

- Determinar qué áreas curriculares participarán. Participarán todas las áreas a excepción del área de Educación Física, porque es un área solo de actividades físico - corporal.

- Cuáles serán procesos de la Institución Educativa a considerar. Los procesos son: Gestión de la Dirección, Gestión de Recursos y el Pedagógico.

- Quienes aplicarán la prueba. La prueba se hará de forma virtual y estará a cargo del docente del Aula de Innovación Tecnológica.

b. Especificar la evaluación. Se defeinen los criterios de evaluación de acuerdo a la Norma ISO 9004 - 2009 [8], adaptando el cuestionario a los procesos de la institución educativa, según las tablas de autoevaluación: a) Elementos claves, y b) Elementos detallados. Descritos en la sección 3 de este artículo.

c. Diseñar la evaluación. Establecer las actividades del plan de evaluación:

- Identificar los aspectos del proceso educativo a considerar en la evaluación: procesos cognitivos y pedagógicos de la sesión de clase, proyectos y unidades de aprendizaje, métodos y técnicas de aprendizaje, recursos tecnológicos.

- Establecer un cronograma de reuniones del equipo del proyecto para tomar las decisiones sobre los aspectos a evaluar.
- Elaborar y evaluar el cuestionario con los indicadores que se encuentran establecidos en los criterios del Modelo de Calidad para la Gestión de las TIC propuesto.

d. Llevar a cabo la evaluación. Se realizará una encuesta virtual, que deberán de responder todos los docentes de la Institución Educativa a excepción de los docentes de Educación Física.

e. Finalizar la Evaluación. se hace un análisis de los resultados para determinar el nivel de madurez para cada criterio de evaluación de las tablas de autoevaluación.

El diagnóstico de la situación actual del sistema de gestión de las TIC en la institución educativa, detectará los criterios que no se cumplen y se propondrán las actividades de mejora. O si los criterios se cumplen se propondrán actividades de mantenimiento.

\subsubsection{Elaboración de los cuestionarios}

Para ejecutar el proceso de autoevaluación se evalúan los criterios de las tablas de autoevaluación, que para el presente estudio se realiza a instituciones educativas peruanas del nivel secundario, son siete tablas de autoevaluación que se están considerando: una tabla para los elementos claves, y 6 tablas para los elementos detallados.

A la vez, se está considerando evaluar las áreas correspondientes a la Gestión de la Dirección, Gestión de los recursos y a los procesos pedagógicos de la institución educativa en evaluación. Mediante encuestas realizadas a los docentes, directivos, y padres de familia, se realiza el proceso de evaluación de los criterios de las siete tablas.

En los cuestionarios, las preguntas corresponden a cada criterio de las tablas, y las posibles respuestas a los indicadores de cada nivel de madurez para cada criterio. Para de esta manera medir si la institución cumple con los criterios evaluados en el contexto de la gestión de las TIC, y determinar el nivel de madurez en los criterios evaluados.

A cada tabla se le asigna como puntaje máximo el número de encuestados por el número de preguntas, y se distribuye el porcentaje de acuerdo a las respuestas de los criterios, en los niveles de madurez de 0 a 5 . Es necesario resaltar que se empieza desde el nivel 0 , que refleja que el criterio no ha alcanzado el nivel 1.

Para determinar en qué nivel se encuentra la institución, todas las tablas tienen que tener 
respuestas en el mismo nivel de madurez. La Tabla 6 resume el número de casos y el porcentaje alcanzado en las encuestas para cada tabla, en cada nivel de madurez.

El Índice de Evaluación (IE) en la Tabla 6 corresponde a:

Total N. ${ }^{\circ}$ Conformidades $\times 100$

$\mathrm{IE} \%=\frac{\text { Puntaje Máximo }}{\text { Pún }}$

$\mathrm{Y}$ el N. ${ }^{\circ}$ Conformidades (NC) es la sumatoria de las opciones marcadas en los criterios del elemento clave o de la sumatoria de las opciones marcadas en los criterios de cada elemento de detallado. Está distribuido en el nivel de madurez en que se encuentra.

\subsubsection{Verificación de las Encuestas (V)}

Para determinar en qué nivel de madurez se encuentra la Institución Educativa, debemos de tomar en cuenta que ningún indicador del mismo nivel de madurez que conforman los criterios de las tablas del modelo debe de quedar vacío. En el caso que un indicador del mismo nivel de madurez quede vacío, se deberá de elaborar las actividades de mejora, y nuevamente se vuelve a iniciar el ciclo del modelo para pasar al siguiente nivel de madurez.
Se sugiere que se realice una encuesta piloto antes de la prueba final, porque permitirá ajustar los resultados del cuestionario final, así la prueba se puede reestructurar o hacer algunos cambios necesarios para la prueba final, el elaborar la prueba piloto queda a decisión del investigador.

\subsubsection{Análisis de Resultados y actividades de Mejora (A)}

- Una vez obtenidos los resultados, se procederá a revisarlos y verificar en qué nivel de madurez se encuentran los criterios del modelo.

- Elaborar las actividades de Mejora (el equipo del proyecto, integrado por los jefes de cada área, realizará reuniones técnico - pedagógicas internamente en sus áreas a fin de revisar los procesos y procedimientos, basándose en los resultados del análisis situacional).

- El equipo directivo revisará los procedimientos una vez terminado.

- El jefe de proyecto capacitará a los docentes en una jornada de capacitación bimestralmente sobre las herramientas TIC que utilizarán y en cómo incorporarlos como estrategias de enseñanza aprendizaje.

- El sistema se empleará durante todo el año lectivo escolar, recopilando la información de las pruebas de progreso que se realizan dos veces al año según norma el Ministerio de Educación.

Tabla 6. Puntajes asignados a las Tablas de Evaluación. Fuente: [Moreno, 2014]

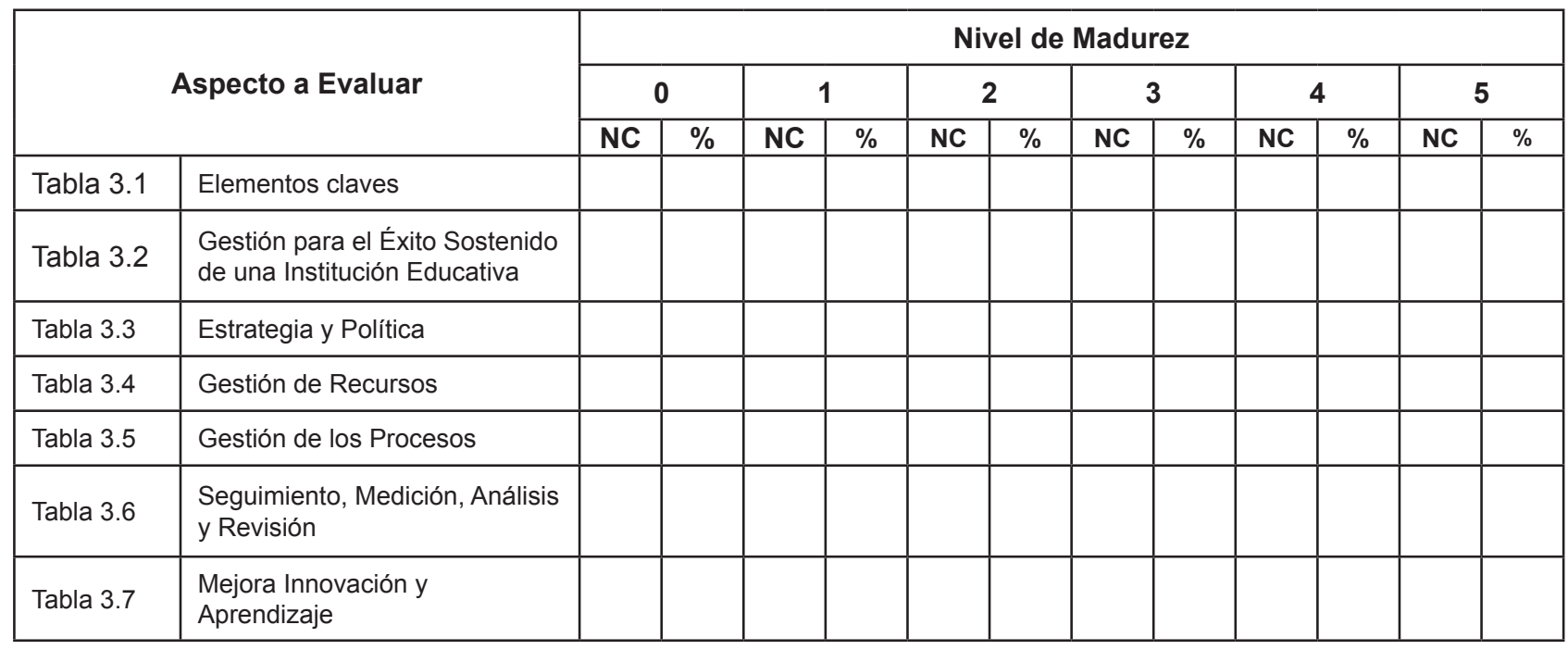




\section{CONCLUSIONES}

El modelo es de gran utilidad para el fortalecimiento del Sistema Educativo, por cuanto aporta herramientas y métodos apropiados para la mejora continua y el establecimiento de una Cultura de la Calidad.

El Modelo desarrollado es un marco de trabajo estructurado por componentes de acuerdo a los procesos del Ciclo de Mejora Continua de W. Deming (PDCA) [5, 9] adaptado para Instituciones Educativas Públicas en la Gestión de las TIC, que ayuda al sistema educativo nacional debido a que puede ser utilizado por otras instituciones educativas que cuenten con una implementación tecnológica adecuada .

Con la implementación del modelo se pretende lograr una mayor satisfacción de las necesidades y expectativas de los alumnos, padres de familia, docentes y demás partes interesadas, porque se ve reflejado en la mejora rendimiento académico de los alumnos.

Finalmente, se ha presentado el Modelo de calidad para la gestión de las TIC; a la vez se ha descrito la herramienta de autoevaluación y se ha presentado detalladamente una guía de implementación del modelo.

\section{REFERENCIAS BIBLIOGRÁFICAS}

[1] Acuña, J. (2012). Modelo de Gestión de Calidad Académica para la Educación Superior, Universidad Latina de Costa Rica.

[2] Bou, C, Escrig, A. (2008). Una evaluación empírica del modelo de excelencia EFQM, España,

[3] Cabero, J. (2008). Creación de una Guía de Evaluación / Autoevaluación de Centros de Recursos Universitarios de Producción de TIC's en la Enseñanza, Revista de medios y educación, ISSN 1133-8482, N. ${ }^{\circ} 32$.

[4] Cuatrecasas L. 2010). Gestión Integral de la Calidad. Barcelona.
[5] Deming W. (1993). The New Economics for Industry, Govermment, Education. Massachusets Institute of Technologies, Center for advance Engineering Study USA Pag. 50.

[6] Fazlildris, K, Anuar, M.(2008). The Impacts of Leadership Style and Best Practices on Company Peformances: Empirical Evidence from Business Firms in Malaysia.

[7] Gasparik, J (2013). Implementation and Evaluation of Model CAF in Education Organizations. Ijonte, International Journal on New Trends in Educations. 22-33.

[8] ISO. Norma Internacional ISO 9004-2009. (2009). Gestión para el Éxito Sostenido de una Organización. Suiza.

[9] McDonnell, L, De Nieves, C. (2006). Comparación entre los Modelos de Gestión de Calidad Total. X Congreso de Ingeniería de Organización. Valencia.

[10]Moreno, J. (2014) Modelo de Calidad para la Gestión de las Tic's en el Proceso de Enseñanza para las Instituciones Educativas Públicas de Educación Secundaria. Facultad de Ingeniería de Sistemas. UNMSM. Lima, Perú.

[11]Piattini M, García, F, de Guzmán I. (2010). Calidad de Sistemas de Información, 2da edic., México.

[12]UNESCO (2008). Estándares de Competencia TIC para Docentes. Francia.

[13]Valanives, N, Angeli C. (2009). Learning and teaching about scientific models with a computer modeling tool, Computers in Human Behavior, 24, 220-233.

[14]Valverde, J. (2010). Enseñar y Aprender con Tecnologías: Un Modelo Teórico para las Buenas Prácticas con TIC. Universidad de Salamanca, 2010, España.

[15]EFQM. (2013). Good Practice Competition, http://www.efqm.org. (Visitado el 1-01.2014) 